\title{
Nasopharyngeal Carcinoma: Current Concepts of Care
}

\author{
Jay S. Cooper
}

Received: 23 April 2012 / Accepted: 25 April 2012 /Published online: 7 June 2012

(C) Springer-Verlag 2012

As a baseline consider the following: in a review of the M.D. Anderson Hospital records from 1948 to 1965, the 5 -year disease-free survival rate for squamous cell carcinomas of the nasopharynx, treated solely by radiation therapy, was $28 \%$ (Fletcher, 2nd ed, p 293). Even for those tumors that had a large benign lymphocytic infiltrate ("lymphoepitheliomas"), the 5-year disease-free survival rate was only $45 \%$. The absence of three-dimensional (3D) imaging, the limitations of hand-calculated radiation planning, the crude (by today's standards) delivery equipment, and the lack of effective systemic agents all contributed to these meager outcomes.

The 1980s gave birth to a revolution. CT scanners started to reveal 3D detail. Computers allowed more complex calculations. Treatment planning simulators and linear accelerators flourished. And chemotherapy began to be seen as both a systemic approach and a biologic modifier of radiation therapy. The North American Intergroup embraced these changes and launched INT 0099 comparing radiation therapy alone versus identical therapy with concurrent cisplatin and added subsequent cisplatin/5-fluorouracil. While not totally without controversy, the superiority of combined therapy seen in this trial caused a paradigm shift that has persisted to date. But, the trial may have raised as many questions as it answered. Are the results of the trial applicable to all populations worldwide? Is combined therapy right for all patients? Does the transition from 2D and 3D radiation therapy to more sophisticated and capable IMRT make the results

J. S. Cooper $(\bowtie)$

Maimonides Cancer Center,

Brooklyn, NY, USA

e-mail: jcooper@maimonidesmed.org of INT 0099 obsolete? Is adjuvant (CDDP/5-FU) needed for all patients? If not, can we triage with certainty?

This issue of the Journal of Radiation Oncology begins with a very personal account by Dr. Al-Sarraf, who served as the study chair of the INT 0099 protocol, describing an intimate "insider's" perspective of the discussions, decisions, and compromises that went into its creation. Drs. Lu and Tham then discuss the implications for patients who have early-stage disease that was excluded from INT 0099. Next Dr. Wang et al. review the effects of the more modern IMRT that simply was not available for use then and reflect on the potential implications of such complex therapy on the proper interpretation of INT 0099 today. Then Dr. Lee et al. present the case for the potential rearrangement in treatment sequence, replacing adjuvant chemotherapy with neo-adjuvant (induction) therapy. This is followed by Dr. Lin's review of the nuances of adjuvant chemotherapy and the possibility of using a marker, such as the plasma EBV titer, as a guide to the necessity of adjuvant therapy in an individual patient. In sum, the manuscripts place the landmark INT 0099 trial in a contemporaneous perspective.

Surely, Medicine will continue its progress and the therapy of nasopharyngeal cancer will continue to evolve. Some future therapy will improve local control, another regional control, and still another distant control. Ironically all are inextricably tied; the improvement of any one makes the importance of the others greater. So, read on for an overview of the current stateof-the-art and a solid foundation for viewing the future.

Jay S. Cooper, M.D.

April 2012 